\title{
Multiple Congenital Arteriovenous Malformations with Involvement of the Vagina and Profuse Hemorrhage from Vaginal Ulcer
}

\begin{tabular}{|l|l|l|}
\hline S.L. & Shashikant L. & Sholapurkar $^{\mathrm{a}}$ \\
\hline S. & Sana & Malhotra $^{\mathrm{a}}$ \\
\hline K. & Kamala & Dhall $^{\mathrm{a}}$ \\
\hline S. & Suman & Kochhar $^{\mathrm{b}}$ \\
\hline
\end{tabular}

aDepartment of Obstetrics and Gynecology, ${ }^{\mathrm{b}}$ Department of Radiodiagnosis, Postgraduate Institute of Medical Education and Research, Chandigarh, India

\section{Key Words}

Arteriovenous malformation

Vaginal ulcer

Pelvic vasculature

Congenital malformations

\section{Abstract}

Congenital arteriovenous malformation (AVM) of the female pelvis is a rare but potentially life-threatening condition. An unusual presentation of AVM involving the vagina, abdominal wall, spleen and thigh, with profuse hemorrhage from a vaginal ulcer is described. Doppler, real time ultrasound and computerized tomography were useful in confirmation and delineation of the extensive AVM in this case. Angiography and embolization were thought risky and possibly unnecessary. Bleeding stopped with bed rest and vaginal packing with gauze soaked in $3 \%$ hypertonic saline. The ulcer healed after 3 months with conservative management. The risk of future problems was explained to the patient and follow-up was advised. Undertreatment of AVM may be preferable to overtreatment, and the aim should be 'control' rather than 'cure'.

Dr. Shashikant L. Sholapurkar, Pool Officer, Department of Obstetrics and Gynecology, P.G.I.M.E.R., Chandigarh-160012 (India)

\section{Introduction}

Congenital arteriovenous malformation (AVM) of the female pelvis is a known clinical entity for more than 100 years now [1]. It is a rare condition, but occasionally leads to life-threatening complications. However, its diagnosis, classification and treatment are still plagued by considerable confusion. It is often the gynecologist who encounters these cases first, but many of them are totally unaware of this condition because of its rarity. This can lead to mistakes in diagnosis and rarely to disastrous consequences due to wrong diagnostic or therapeutic interventions. A rare case of pelvic AVM presenting with bleeding from vaginal ulcer is described. Clinical presentation, diagnosis and management of this uncommon condition are discussed with emphasis on the gynecological perspective.

\section{Case Report}

Mrs. R.K., a 43-year-old multiparous lady, attended the gynecological outpatient department with profuse vaginal bleeding for more than 20 days and prolapse of vaginal mucosa for 10 years. She had received 4 units of compatible blood before coming to this hospital. Her menstrual cycles were regular with average flow for 4-5 days without any pain, and her last 
menstrual period was 25 days ago. She had no postcoital bleeding or trauma. There were no bladder or bowel problems and no major illness in the past. She was married for 18 years and had 3 full-term uneventful pregnancies and normal vaginal deliveries in a hospital. She also had an induced abortion at 8 weeks in her third pregnancy 11 years ago, without any complication. All children were well and the last childbirth was 9 years ago. She had tubal ligation by minilaparotomy 6 years ago in a civil dispensary, and no records were available. None of her family members had similar illness or any physical deformities.

On examination, she had severe pallor and blood pressure was 100/60 mm Hg. There were no signs of hyperdynamic circulation or cardiac failure. There was no cardiomegaly or any murmur. Chest was clear. Abdominal examination revealed a mass of dilated blood vessels occupying an area of $10 \times 6 \mathrm{~cm}$ in the subcutaneous tissue of the right iliac fossa, extending into the flank. The mass was compressible and filled up slowly when pressure was released. She had a large

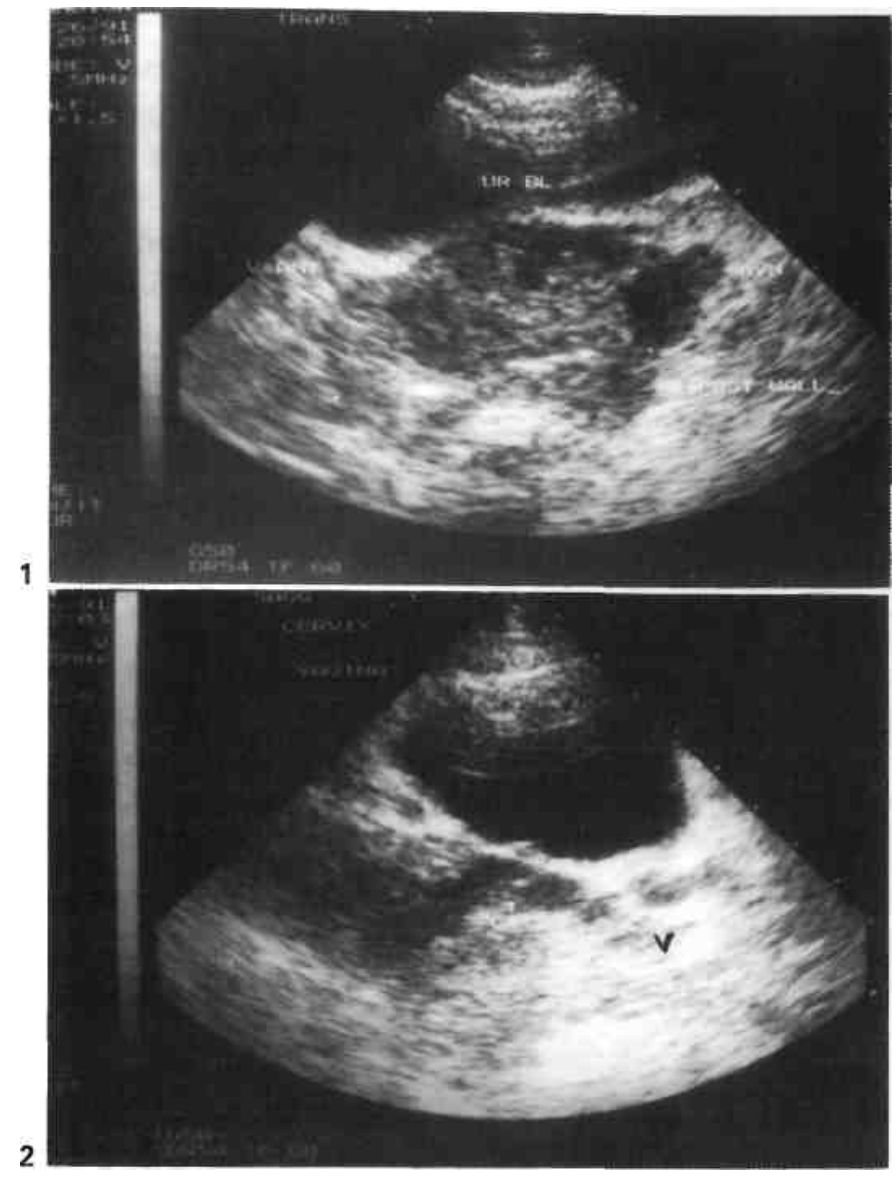



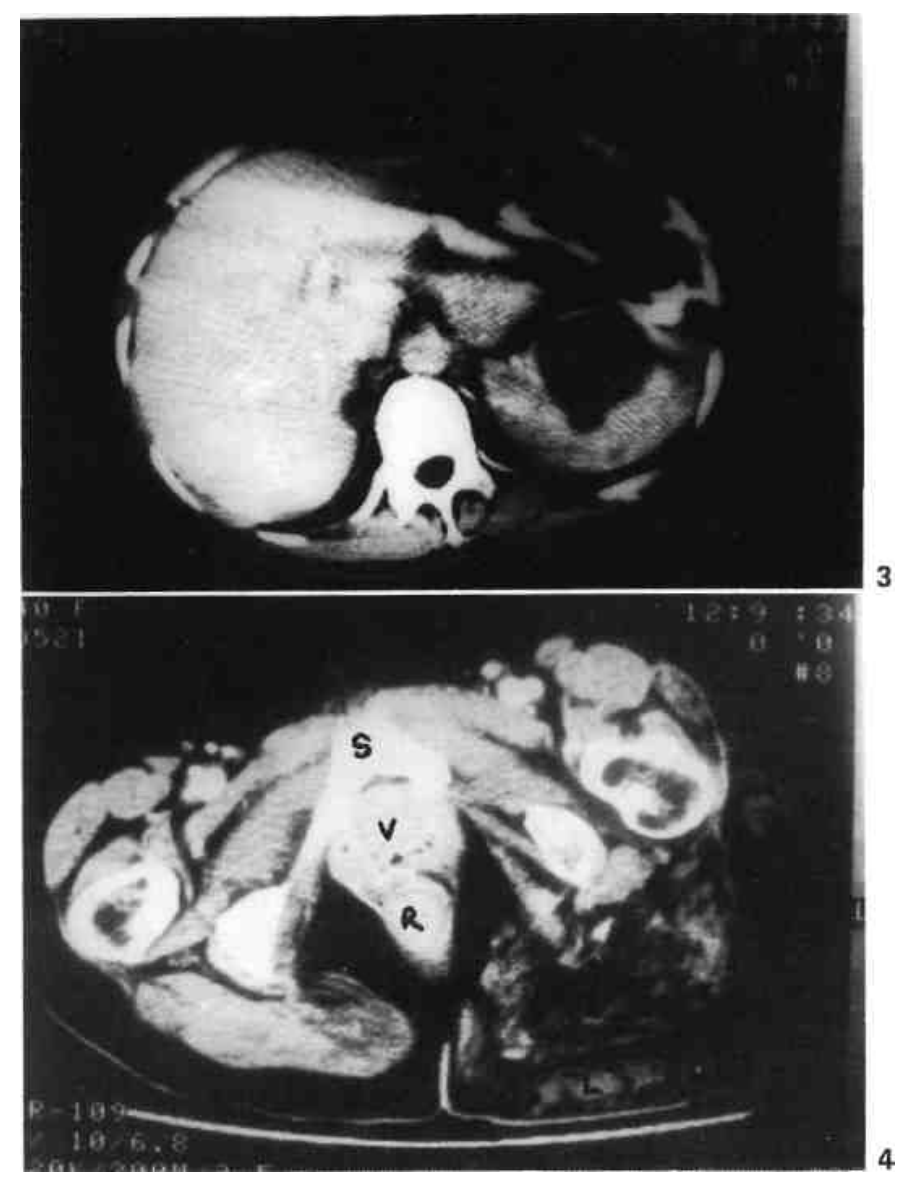

Fig. 1, 2. Ultrasound appearance of AVM involving the vagina. 1 Transverse scan showing multiple linear and round hypoechoic shadows in the anterior and posterior vaginal walls with markedly increased thickness. 2 Longitudinal scan showing multiple hypoechoic areas in the vaginal walls $(\mathrm{V})$.

Fig. 3. CT (without contrast injection) showing splenic AVM.

Fig. 4. CT of the pelvis with intravenous injection of contrast medium (cross-sectional view) showing the vagina $(V)$ engorged with blood. $\mathrm{S}=$ Symphysis pubis; $\mathrm{R}=$ rectum; $\mathrm{L}=$ lipoma on the left buttock.

lipoma on the left gluteal region and diluted, tortuous, venous channels on the posterior aspect of the left thigh. Both her feet were malformed with multiple chondromas involving the toes. On specific questioning, she revealed that she had these deformities right from childhood, but they did not create problems at any stage.

External genitalia were normal. Anterior and posterior vaginal walls were prolapsed out of the introitus, purple in color and engorged with blood. There was no cough impulse. On palpation, they were nonpulsatile but compressible and refilled with blood slowly. There was a shallow ulcer of $2 \times 2 \mathrm{~cm}$ on the posterior vaginal wall just above the introitus. Its margins were raised, the floor was not indurated and the ulcer bled on touch. Cervix was also congested, and there was no abnormal discharge. Vaginal examination revealed a multiparous size, midposed, mobile uterus. There was a feeling of multiple cordlike structures in both lateral fornices, but no 
pulsation could be made out. Rectal examination revealed absence of rectocele. A diagnosis of multiple AVM involving the pelvis, vagina, left thigh and abdominal wall was made, and she was admitted to the ward.

Further investigations revealed $\mathrm{Hb}$ of $5 \mathrm{~g} / \mathrm{dl}$ and normal urinaly-sis, blood urea, creatinine and plasma sugars. Chest X-ray did not

reveal cardiomegaly, and EKG was normal. Abdominopelvic ultrasound showed a hypoechoic area in the spleen measuring $48 \times 50 \mathrm{~mm}$ and marked increase in thickness of vaginal walls with presence of multiple linear and round hypoechoic areas suggestive of AVM (fig. 1 , 2). Other organs were normal. Doppler examination revealed slow flow pattern and absence of significant shunting. Computerized tomogram of the abdomen and pelvis showed a hypoechoic area in spleen suggestive of AVM (fig. 3). It also showed that the whole length of the vagina was involved by the AVM (fig. 4). Uterus and adnexa were normal. MRI was not available. After admission, she was built up with blood transfusions and advised bed rest with the foot end of the bed raised. Gentle vaginal packing was done with a gauze soaked in 3\% hypertonic saline, which was changed daily. While in the ward, she continued to have bouts of vaginal bleeding some of which were profuse. Fortunately, bleeding used to cease spontaneously with rest and packing. Six units of compatible blood were transfused. After 1 week, bleeding stopped completely. Further management was planned in consultation with vascular surgeons, plastic surgeons and radiologists. The nature and extent of the disease was explained to the patient. Angiography and

127

therapeutic embolization were thought to be risky because of the extensive nature of the lesion. These were also thought to be unnecessary unless the ulcer failed to heal or bleeding continued. This was also discussed with the patient. With conservative management, the ulcer started healing, and she was discharged after 15 days with advice to continue the same treatment at home. Abstinence from coitus was also advised. Three months later, the ulcer had completely healed. She had had occasional coitus with the husband without any bleeding. The risk of bleeding due to coitus was thought to be low as it had never occurred before. She agreed for follow-up every 6 months or in case of some problem.

Discussion

An AVM consists of a proliferation of arterial and venous channels with fistula formation and admixture of small capillary-like channels. They can be either congenital or acquired [2]. Congenital AVMs are thought to be present from birth and differ from hemangiomas. They also should not be confused with varicosities of vulva, vagina and lower extremities occurring during pregnancy. These venous dilatations are because of hormonal influence and pressure by the gravid uterus and disappear spontaneously after delivery.

Congenital AVMs generally involve multiple sites and can become symptomatic at any age. Many of these cases may be totally asymptomatic. Others have two types of manifestations: systemic and/or local [3]. Systemic manifestations result from shunting of large quantities of blood leading to hyperdynamic circulation, cardiomegaly and eventually cardiac failure. Local manifestations include physical deformity, pain, ulceration, bleeding and loss of function. In gynecological practice, the majority of pelvic and uterine AVMs present with menorrhagia, metror-rhagia or postmenopausal bleeding. Pelvic AVM leading to vaginal ulcer and profuse hemorrhage, as seen in the present case, has not been previously described in the literature. Long-standing engorgement of vaginal walls with blood probably resulted in ulcer formation in this case, not unlike varicose ulcers. 
Diagnosis of this rare condition requires a high degree of awareness. Also, additional investigations are neces-

sary to conñrm the nature and true extent of the lesion. However, when AVM is suspected, a biopsy or curettage is contraindicated because of risk of massive hemorrhage. In the past, angiography was the mainstay of diagnosis, which is an invasive technique and not without risks of its own. Of late, noninvasive techniques like Doppler, real time ultrasound, CT and MRI are gaining more importance in diagnosis and evaluation of AVMs [1,3]. Angiography should be reserved for cases where intervention is planned.

The treatment of pelvic AVM requires careful planning, a multidisciplinary approach, facilities of intensive care and access to modern imaging technology. Treatment itself can be hazardous, and results are unpredictable at present, and this should be explained to the patient [1]. Asymtpomatic cases should be left alone. Transcath-eter embolization of involved vessels with particulate substances is the treatment of choice in the majority of AVMs, which should also allow preservation of fertility in younger women [4]. In older women, hysterectomy may be performed with or without embolization if the uterus is the site of bleeding. Ligation of multiple feeding vessels is not recommended for congenital pelvic AVM [ 1 ].

The present case had multiple AVMs as well as other soft tissue anomalies, like lipoma and chondromas. However, classifying these cases into various syndromes has not been found useful in clinical practice as these are widely overlapping heterogenous abnormalities [3]. Real time ultrasonography and CT scan showed the extensive nature of AVM in this case (fig. 1-4). The patient was symptomatic only for the ulcer, and bleeding stopped with rest and packing. Angiography and embolization were thought to be risky and possibly unnecessary. The ulcer healed after 3 months with conservative management. Risk of bleeding with coitus was thought to be low as it had never occurred before. This case highlights the principle that undertreatment may be preferable to overtreatment, and the aim of the treatment should be 'control' rather than 'cure' [3]. This patient will be kept under life-long follow-up to watch for the development of any other symptoms or complications. In such an event, embolization would be the most suitable treatment for her pelvic AVM.

References

Beller U, Rosen RJ, Beckman EM, Markoff G, Berenstein A: Congenital arteriovenous malformation of the female pelvis: A gynecologic perspective. Am J Obstet Gynecol 1988; 159: 1153-1160.

Fleming H, Ostor AG, Pickel H, Fortune DW: Arteriovenous malformations of the uterus. Obstet Gynecol 1989;73:209-213. Halliday AW, Mansfield AO: Arteriovenous malformations: Current management approaches. Br J Hosp Med 1989;42:196-202.

Poppe W, Van Assche FA, Wilms G, Favril A, Baert A: Pregnancy after transcatheter embolisation of a uterine arteriovenous malformation. Am J Obstet Gynecol 1987; 156:1179-

128

Sholapurkar/Malhotra/Dhall/Kochhar $\quad$ AVM of the Pelvis 